\title{
Misurare la resistenza scheletrica oltre la DEXA
}

\author{
Davide Diacinti ${ }^{1,2} \cdot$ Salvatore Minisola ${ }^{3}$
}

Accettato: 30 marzo 2020 / Pubblicato online: 25 maggio 2021

(c) The Author(s) 2021

L'osteoporosi è caratterizzata da una riduzione della massa ossea e da alterazioni della microarchitettura, in particolare dell'osso spongioso, ma anche dell'osso compatto. Sia la quantità che la qualità dell'osso sono determinanti della sua resistenza. La quantità dipende dalla densità ossea che può essere valutata con la tecnica Dual-X-ray Absorptiometry (DXA), misurando la Bone Mineral Density (BMD). La qualità dell'osso dipende da diversi fattori: velocità del turnover osseo, distribuzione della massa ossea, microfratture, grado e qualità della mineralizzazione, qualità delle fibre di collageno e microarchitettura ossea, soprattutto a livello trabecolare. Con la tecnica DXA è possibile ottenere una valutazione indiretta della microarchitettura ossea per ogni corpo vertebrale lombare applicando un algoritmo denominato Trabecular Bone Score (TBS), che rianalizza la dinamica spaziale delle variazioni dell'intensità dei pixel sul tratto vertebrale lombare, ricreando una mappatura qualitativa trabecolare. I valori di riferimento per donne in post menopausa, stabiliti per analogia con le tre categorie della BMD, ovvero massa ossea normale, osteopenia e osteoporosi sono: TBS $\geq 1.350$ indice di una microarchitettura normale; TBS tra 1.200 e 1.350: microarchitettura parzialmente degradata; TBS $\leq 1.200$ : microarchitettura altamente degradata [1].

È stata dimostrata la validità del TBS quale indice di rischio fratturativo in popolazioni con alterata qualità ossea come, ad esempio, in pazienti con insufficienza renale [2].

$凶$ S. Minisola

salvatore.minisola@uniroma1.it

1 Dipartimento di Scienze Odontostomatologiche e Maxillo Facciali, Università Sapienza, Roma, Italia

2 Dipartimento di Diagnostica Radiologica, Fondazione Policlinico e Università Tor Vergata, Roma, Italia

3 Dipartimento di Scienze Cliniche Internistiche, Anestesiologiche e Cardiovascolari, Università Sapienza, Roma, Italia
Negli ultimi anni sono state sviluppate nuove tecniche di imaging ad alta risoluzione per la valutazione diretta non invasiva della microarchitettura trabecolare: la tomografia computerizzata (HRCT e micro-TC effettuate rispettivamente a livello delle vertebre e del radio distale-falangi della mano) e la risonanza magnetica (HR-MRI o $\mu$-MRI effettuata a livello di tibia distale, calcagno e polso). Recentemente, con lo sviluppo di TC multistrato (Fig. 1) è stata

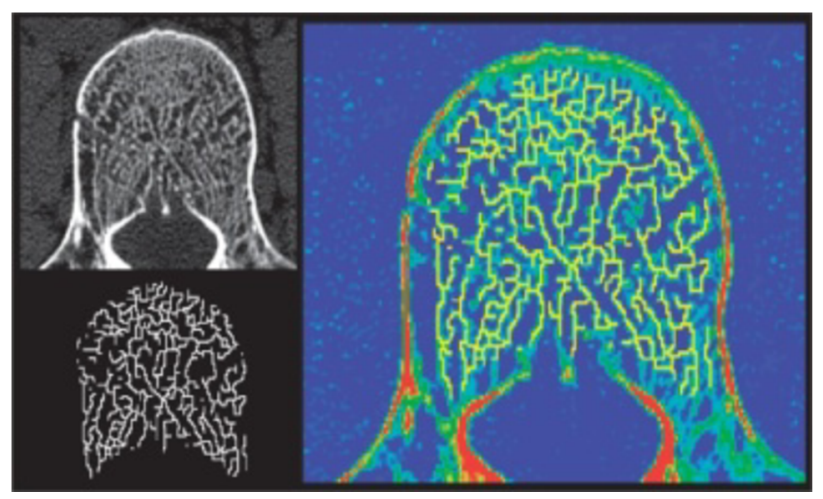

Fig. 1 Valutazione della trabecolatura ossea vertebrale mediante *TC multistrato *(MDCT)

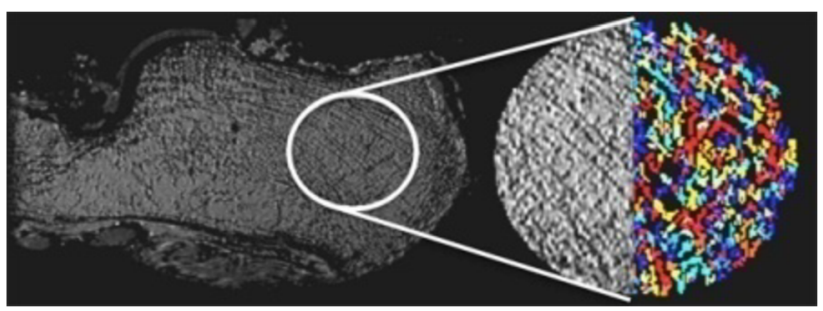

Fig. 2 Valutazione dell'architettura dell'osso trabecolare tramite risonanza magnetica ad alta risoluzione $*($ HR-MRI $) *$ a livello del calcagno 

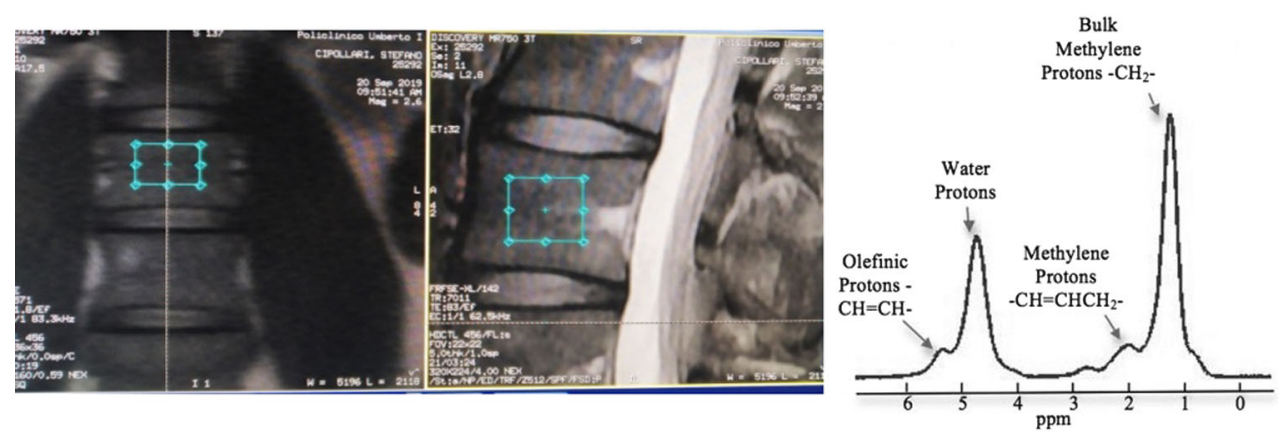

Fig. 3 *Spettroscopia con Risonanza Magnetica **(1H-MRS) *applicata a livello L1-L4 con box posizionato su uno dei somi vertebrali in esame, applicando sequenze PRESS; esempio di un grafico di spettro- scopia a livello lombare in cui si documentano 4 diversi picchi: grassi saturi a 1,3 ppm, grassi residui a 2,01 ppm, grassi insaturi a 5,3 ppm e i protoni dell'acqua a $4,7 \mathrm{ppm}$ raggiunta una risoluzione spaziale pari a $300 \mu \mathrm{m}$ rispetto a $0,6 \mathrm{~mm}$ dell'HRCT, sufficiente per valutare lo spazio trabecolare e le connettività delle trabecole, evidenziando la capacità nell'analisi strutturale dell'osso e discriminando soggetti con e senza fratture vertebrali con maggior significatività rispetto alla BMD valutata con la DXA: pertanto, utile nel predire l'insorgenza di future fratture vertebrali [3]. Anche con l'RM è possibile valutare l'architettura dell'osso trabecolare tramite HR-MRI (Fig. 2) o $\mu$-MRI, poiché è stato dimostrato che il $\mathrm{T}^{*}$ del midollo osseo è influenzato non solo dalla densità trabecolare, ma anche dalla sua architettura spaziale, risultando differente nel midollo con numerose e sottili trabecole rispetto a quello con poche e spesse trabecole, in presenza di uguale densità ossea [4]. L'RM permette di valutare la qualità dell'osso trabecolare calcolando alcuni parametri isto-morfometrici standard della struttura ossea: frazione di area trabecolare ossea, spessore delle trabecole e spazio intertrabecolare. Poiché le trabecole ossee sono molto sottili (da 50 a $200 \mu \mathrm{m}$ ), è necessario avere immagini a elevata risoluzione spaziale. Ciò è difficile da ottenere nello scheletro assiale (vertebre o femore prossimale), per cui l'HR-MRI viene effettuata su tibia distale, calcagno e polso e i parametri della struttura trabecolare valutati con HR-MRI correlano in maniera significativa con l'età e i valori della densitometria ossea; in particolare, lo spessore trabecolare, la frazione trabecolare e il numero delle trabecole diminuiscono, mentre lo spazio intertrabecolare aumenta con la riduzione della densità minerale ossea [5]. Una recente metodica di imaging utilizzata per la valutare indirettamente la resistenza scheletrica è la risonanza magnetica spettroscopica (1H-MRS) applicata a livello L1-L4 con posizionamento di un box su uno dei somi vertebrali in esame, applicando sequenze PRESS; la successiva elaborazione dei dati permette di ottenere spettri relativi al contenuto di grassi e acqua all'interno della vertebra e di calcolare la quantità di grasso midollare (Bone Marrow Fat, BMF) (Fig. 3). In pazienti osteoporotiche in età post-menopausale è emerso come un aumento dei valori di BMF sia correlato a un basso valore di BMD e aumentata prevalenza di fratture ossee, evidenziando che elevati valori di BMF possano essere indici di possibile fragilità scheletrica [6].

Funding Note Open access funding provided by Università degli Studi di Roma La Sapienza within the CRUI-CARE Agreement.

Conflitto di interesse Gli autori Davide Diacinti e Salvatore Minisola dichiarano di non avere conflitti di interesse.

Consenso informato Lo studio presentato in questo articolo non ha richiesto sperimentazione umana.

Studi sugli animali Gli autori di questo articolo non hanno eseguito studi sugli animali.

Nota della casa editrice Springer Nature rimane neutrale in riguardo alle rivendicazioni giurisdizionali nelle mappe pubblicate e nelle affiliazioni istituzionali.

Open Access This article is licensed under a Creative Commons Attribution 4.0 International License, which permits use, sharing, adaptation, distribution and reproduction in any medium or format, as long as you give appropriate credit to the original author(s) and the source, provide a link to the Creative Commons licence, and indicate if changes were made. The images or other third party material in this article are included in the article's Creative Commons licence, unless indicated otherwise in a credit line to the material. If material is not included in the article's Creative Commons licence and your intended use is not permitted by statutory regulation or exceeds the permitted use, you will need to obtain permission directly from the copyright holder. To view a copy of this licence, visit http://creativecommons.org/licenses/by/4.0/.

\section{Bibliografia}

1. Hans D, Goertzen AL, Krieg M-A, Leslie WD (2011) Bone microarchitecture assessed by TBS predicts osteoporotic fractures independent of bone density: the Manitoba study. J Bone Miner Res 26:2762-2769

2. Naylor KL, Lix LM, Hans D et al (2016) Trabecular bone score in kidney transplant recipients. Osteoporos Int 27(3):1115-1121

3. Ito M, Ikeda K, Nishiguchi M et al (2005) Multi-detector row CT imaging of vertebral microstructure for evaluation of fracture risk. J Bone Miner Res 20(10):1828-1836 
4. Benito M, Gomberg B, Wehrli FW et al (2003) Deterioration of trabecular architecture in hypogonadal men. J Clin Endocrinol Metab 88:1497-1502

5. Ouyang X, Selby K, Lang P et al (1997) High resolution MR imaging of the calcaneus: age-related changes in trabecular struc- ture and comparison with DXA measurements. Calcif Tissue Int 60:139-147

6. Patsch JM, Li X, Baum T et al (2013) Bone marrow fat composition as a novel imaging biomarker in postmenopausal women with prevalent fragility fractures. J Bone Miner Res 28(8):1721-1728 